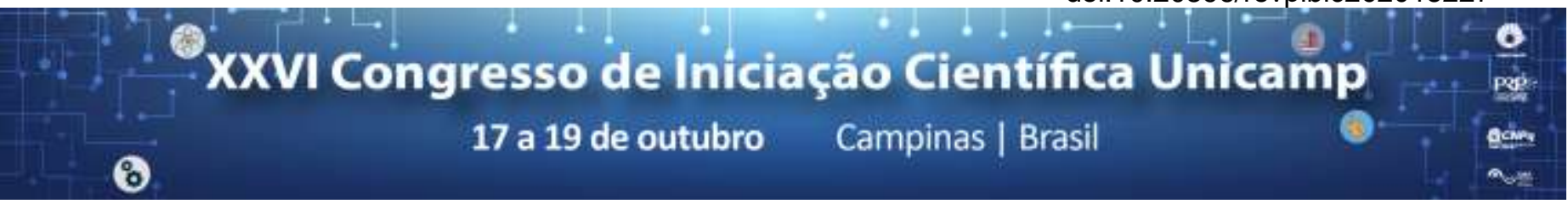

\title{
Tradução e adaptação cultural do "Illness Perception Questionnaire-Revised for Dental" para uso em pais/responsáveis por crianças com Cárie Precoce da Infância
}

\section{Bruna L. Basso*, Taís S. Barbosa.}

\section{Resumo}

A cárie dentária é uma doença crônica que necessita de estratégias adequadas de autoRregulação; no entanto, no Brasil, ainda não há um instrumento específico para avaliar a representação/percepção cognitiva e emocional dos pais/responsáveis por crianças com cárie dentária. Neste estudo, o questionário "Illness Perception QuestionnaireRevised for Dental" (IPQ-RD) foi traduzido, retraduzido e culturalmente adaptado para uso na população brasileira.

\section{Palavras-chave: Cárie dentária, Questionários, Tradução (Processo).}

\section{Introdução}

É importante que os pais/responsáveis, como principais cuidadores da saúde bucal da criança, apresentem a correta representação da doença, que varia do modelo desorganizado (fase aguda - presença da doença somente quando há sintomas) para o modelo organizado (fase crônica - presença da doença mesmo na ausência de sintomas), para adesão efetiva em estratégias de autorregulação da doença. ${ }^{1}$ Neste contexto, o questionário "Illness Perception Questionnaire-Revised for Dental" (IPQ-RD) foi desenvolvido na língua inglesa para avaliar a representação/percepção cognitiva e emocional dos pais/responsáveis por crianças com cárie dentária. ${ }^{2}$ Este estudo objetivou traduzir e adaptar culturalmente o IPQ-RD para o Português Brasileiro.

\section{Resultados e Discussão}

O IPQ-RD consiste de 32 itens divididos em dimensões cognitivas e representações sociais, com opções de resposta em escala Likert de 5 pontos: concordo totalmente (escore 1) a discordo totalmente (escore 5). ${ }^{2}$ Quanto maior escore, menor a percepção da doença.

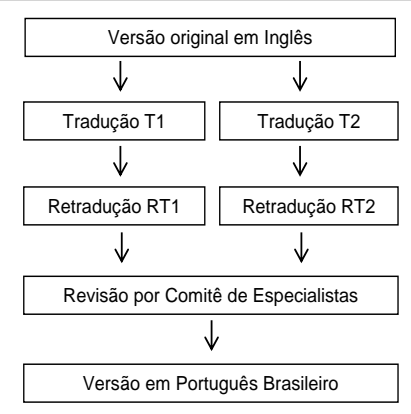

Figura. Resumo do processo de tradução e adaptação cultural (IPQ-RD). ${ }^{3}$

Tabela. Sinopse do processo de decisão relativo à especificação da versão em Português Brasileiro para construção do instrumento final.

\begin{tabular}{|c|c|c|c|c|c|}
\hline & Termos & Tradução & Comitê Revisor & Retradução & $\begin{array}{l}\text { Comitê } \\
\text { Revisor }\end{array}$ \\
\hline Título & IIIIness / Dental & T3 & Doença / Odontologia & $\mathrm{VO}=\mathrm{RT} 1 \approx \mathrm{RT} 2$ & \\
\hline $\begin{array}{l}\text { Q1/Q2 } \\
\text { /Q19 }\end{array}$ & $\begin{array}{l}\text { Cavities } \\
\text { decay) }\end{array}$ & $\mathrm{T} 1 \approx \mathrm{T} 2$ & Cárie dentária & $\mathrm{VO}=\mathrm{RT} 1 \approx \mathrm{RT} 2$ & \\
\hline Q3 & Cavities & $\mathrm{T} 1 \approx \underline{\mathrm{T} 2}$ & Cárie dentária & $\mathrm{VO}=\mathrm{RT} 1 \approx \mathrm{RT} 2$ & \\
\hline $\begin{array}{l}\text { Q4/Q5 } \\
\text { /Q6 }\end{array}$ & \begin{tabular}{|l|} 
Cavities I has \\
had a big effect
\end{tabular} & T3 & $\begin{array}{l}\text { Cárie dentária / tem } \\
\text { comprometido muito }\end{array}$ & $\mathrm{VO}=\mathrm{RT} 1 \approx \mathrm{RT} 2$ & \\
\hline Q7 & $\begin{array}{l}\text { Cavities / has } \\
\text { had a big effect / } \\
\text { school work. }\end{array}$ & T3 & $\begin{array}{l}\text { Cárie dentária / tem } \\
\text { comprometido muito / } \\
\text { desempenho escolar }\end{array}$ & $\mathrm{VO} \approx \mathrm{RT} 1 \neq \mathrm{RT} 2$ & \\
\hline Q8 & $\begin{array}{l}\text { Cavities I has } \\
\text { had a big effect }\end{array}$ & T3 & $\begin{array}{l}\text { Cárie dentária / tem } \\
\text { comprometido muito }\end{array}$ & VO $\approx R T 1 \neq R T 2$ & \\
\hline Q9 & Cavities & $\mathrm{T} 1=\mathrm{T} 2$ & Cárie dentária & VO $\approx \mathrm{RT} 1 \approx \mathrm{RT} 2$ & \\
\hline Q10 & Cavities & $\mathrm{T} 1=\mathrm{T} 2$ & Cárie dentária & $\mathrm{VO}=\mathrm{RT} 1 \approx \mathrm{RT} 2$ & $\begin{array}{l}\text { Compromete } \\
\text { muito }\end{array}$ \\
\hline
\end{tabular}

\begin{tabular}{|c|c|c|c|c|c|}
\hline & Termos & Tradução & Comitê Revisor & Retradução & Comitê Revisor \\
\hline Q11 & $\begin{array}{l}\text { I cannot easily live / } \\
\text { cavities }\end{array}$ & T3 & $\begin{array}{l}\text { Eu não lido bem / } \\
\text { cárie dentária }\end{array}$ & $\mathrm{VO}=\mathrm{RT} 1 \neq \mathrm{R} T 2$ & $\begin{array}{ll}\begin{array}{l}\text { Não } \\
\text { facilmente }\end{array} & \text { vivo } \\
\end{array}$ \\
\hline Q12 & $\begin{array}{l}\text { Cavities / has a big } \\
\text { effect on how } \\
\text { others see me }\end{array}$ & T3 & $\begin{array}{l}\text { Cárie dentária / tem } \\
\text { grande impacto na } \\
\text { forma como os outros } \\
\text { me veem }\end{array}$ & $\mathrm{VO}=\mathrm{RT} 1 \approx \mathrm{RT} 2$ & $\begin{array}{l}\text { Compromete } \\
\text { muito }\end{array}$ \\
\hline Q13 & $\begin{array}{|ll|}\begin{array}{l}\text { Cavities } \\
\text { caused }\end{array} & \text { has } \\
\end{array}$ & T3 & $\begin{array}{l}\text { Cárie dentária / tem } \\
\text { me causado }\end{array}$ & VO $\approx R T 1 \approx R T 2$ & \\
\hline Q14 & $\begin{array}{l}\text { Cavities / has had a } \\
\text { big effect }\end{array}$ & T3 & $\begin{array}{l}\text { Cárie dentária / tem } \\
\text { comprometido muito }\end{array}$ & $\mathrm{VO}=\mathrm{RT} 1 \approx \mathrm{T} 2$ & \\
\hline Q15 & $\begin{array}{l}\text { To improve the } \\
\text { cavities }\end{array}$ & T3 & $\begin{array}{lll}\begin{array}{l}\text { Controlar a cárie } \\
\text { dentária }\end{array} & \\
\end{array}$ & $\mathrm{VO} \approx \mathrm{RT} 1 \approx \mathrm{T} 2$ & \\
\hline Q16 & \begin{tabular}{|l|} 
Make his/her \\
cavities better.
\end{tabular} & T3 & $\begin{array}{lll}\begin{array}{l}\text { Controlar ar a } \\
\text { dentária. }\end{array} & \\
\end{array}$ & $\begin{array}{l}\mathrm{VO} \approx \mathrm{RT} 1 \approx \mathrm{RT} 2 \\
\end{array}$ & \begin{tabular}{|l|} 
Tratamento \\
odontológico
\end{tabular} \\
\hline Q17 & 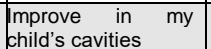 & T3 & \begin{tabular}{|lll}
$\begin{array}{c}\text { Controle } \\
\text { dentária }\end{array}$ & da & cárie \\
\end{tabular} & VO $\approx R T 1 \approx R T 2$ & \\
\hline Q18 & $\begin{array}{l}\text { What my child does } \\
\text { decides / cavities }\end{array}$ & T3 & $\begin{array}{l}\text { As escolhas / cárie } \\
\text { dentária. }\end{array}$ & VOFRT1FRT2 & \\
\hline Q20 & $\begin{array}{l}\text { What I do decides / } \\
\text { cavities }\end{array}$ & T3 & $\begin{array}{l}\text { As minhas escolhas } \\
\text { são decisivas / cárie } \\
\text { dentária }\end{array}$ & VO\#RT1キRT2 & \\
\hline$\overline{Q 21}$ & $\begin{array}{l}\text { To stop my child's } \\
\text { cavities }\end{array}$ & T3 & \begin{tabular}{|lll}
$\begin{array}{l}\text { Paralisar } \\
\text { dentária }\end{array}$ & a & cárie \\
\end{tabular} & $\mathrm{VO}=\mathrm{RT} 1 \approx \mathrm{T} 2$ & \\
\hline Q22 & Cavities & $\mathrm{T} 1 \approx \underline{\mathrm{T} 2}$ & Cárie dentária & $\mathrm{VO}=\mathrm{RT} 1 \approx \mathrm{RT} 2$ & \\
\hline Q23 & $\begin{array}{l}\text { Cavities / will not } \\
\text { get better }\end{array}$ & T3 & $\begin{array}{l}\text { Cárie dentária / não } \\
\text { será controlada }\end{array}$ & $\mathrm{VO}=\mathrm{RT} 1 \approx \mathrm{RT} 2$ & \\
\hline Q24 & $\begin{array}{l}\text { Cavities / likely to } \\
\text { be long-lasting // } \\
\text { short-term }\end{array}$ & T3 & $\begin{array}{l}\text { Cárie dentária / } \\
\text { provavelmente terá } \\
\text { longa durabilidade / } \\
\text { curta durabilidade. }\end{array}$ & $\mathrm{VO}=\mathrm{RT} 1 \neq \mathrm{RT} 2$ & \\
\hline Q26 & $\begin{array}{l}\text { There will be times / } \\
\text { cavity }\end{array}$ & T3 & $\begin{array}{l}\text { Haverá momentos / } \\
\text { cárie dentária }\end{array}$ & $\mathrm{VO}=\mathrm{RT} 1 \neq \mathrm{RT} 2$ & \\
\hline Q27 & $\begin{array}{l}\text { Clear } \\
\text { understanding }\end{array}$ & T3 & Boa compreensão & $\mathrm{VO}=\mathrm{RT} 1 \approx \mathrm{RT} 2$ & \\
\hline Q28 & Cavity & T2 & Cárie dentária & $\mathrm{VO}=\mathrm{RT} 1 \approx \mathrm{RT} 2$ & \\
\hline Q29 & $\begin{array}{|lr|}\begin{array}{l}\text { Upset when I think } \\
\text { about my } \\
\text { suffering }\end{array} & \text { child } \\
\text { cavities } & \text { from } \\
\end{array}$ & T3 & $\begin{array}{l}\text { Chateado quando eu } \\
\text { penso sobre a cárie } \\
\text { dentária }\end{array}$ & $\mathrm{VO}=\mathrm{RT} 1 \approx \mathrm{RT} 2$ & \\
\hline Q30 & Cavities & $\mathrm{T} 1=\mathrm{T} 2$ & Cárie dentária & $\mathrm{VO}=\mathrm{RT} 1 \approx \mathrm{RT} 2$ & \\
\hline Q31 & $\begin{array}{l}\text { Cavities makes me } \\
\text { feel angry. }\end{array}$ & T1 $\approx \mathrm{T} 2$ & $\begin{array}{l}\text { Cárie dentária faz me } \\
\text { sentir zangado. }\end{array}$ & VO=RT1 RT2 & \\
\hline Q32 & Cavities & $\mathrm{T} 1=\mathrm{T} 2$ & Cárie dentária & $\mathrm{VO}=\mathrm{RT} 1 \neq \mathrm{RT} 2$ & \\
\hline $\begin{array}{l}\text { Answ } \\
\text { ers }\end{array}$ & \begin{tabular}{|l|} 
Strongly agree \\
Neither agree nor \\
disagree \\
Strongly disagree \\
\end{tabular} & $\underline{T 1} \approx \mathrm{T} 2$ & $\begin{array}{l}\text { Concordo totalmente } \\
\text { Não concordo, nem } \\
\text { discordo } \\
\text { Discordo totalmente }\end{array}$ & $\mathrm{VO}=\mathrm{RT} 1 \approx \mathrm{RT} 2$ & \\
\hline \multicolumn{6}{|c|}{ 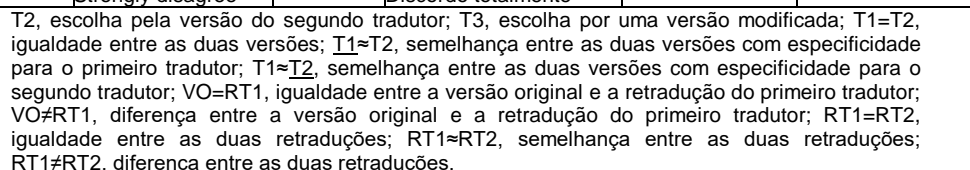 } \\
\hline
\end{tabular}

\section{Conclusões}

A versão em Português Brasileiro do IPQ-RD mostrou ser de fácil compreensão pelos adolescentes.

\section{Agradecimentos}

Aos tradutores, membros do Comitê Revisor e PIBIC/CNPq.

${ }^{1}$ Edgar, K.A.; Skinner, T.C. Illness representations and coping as predictors of emotional

well-being in adolescents with type 1 diabetes. J Pediatr Psychol. 2003,28,485-493.

${ }^{2}$ Nelson, S.; Slusar, M.B.; Albert, J.M.; Liu, Y.; Riedy, C.A. Psychometric properties of a caregiver illness perception measure for caries in children under 6 years old. J Psychosom Res. 2016,81:46-53.

${ }^{3}$ Guillemin, F.; Bombardier, C.; Beaton, D. Cross-cultural adaptation of health-related quality of life measures: literature review and proposed guidelines. J Clin Epidemiol. 1993,46,141732 . 